\title{
New Trends in Nanotribology
}

\author{
O. Gulseren - N. Manini $\cdot$ E. Meyer $\cdot$ \\ E. Tosatti $\cdot$ M. Urbakh $\cdot$ A. Vanossi
}

Published online: 10 August 2010

(C) Springer Science+Business Media, LLC 2010

This issue of Tribology Letters contains articles presented at a recent conference "Trends in Nanotribology" which took place in Leonardo Da Vinci Building of the International Centre for Theoretical Physics in Trieste on October 2010. The conference was part of activities coordinated under the guidance of the European Science Foundation, where groups from 16 different European countries collaborate in the ESF-FANAS-programme and try to answer some of the fundamental questions of Tribology.

The old field of friction, started by Leonardo Da Vinci, entered in an exciting new phase, where novel regimes are being discovered. Superlubricity, wearless sliding, control of friction, and manipulation of nanoparticles are being

O. Gulseren

Department of Physics, Bilkent University, 06800 Ankara, Turkey

N. Manini

Dipartimento di Fisica, Universita degli Studi di Milano,

Via Celoria 16, 20133 Milan, Italy

E. Meyer

Department of Physics, University of Basel,

Klingelbergstrasse 82, 4056 Basel, Switzerland

E. Tosatti - A. Vanossi

International School for Advanced Studies (SISSA)

and CNR-IOM DEMOCRITOS Simulation Center,

Via Bonomea 265, 34136 Trieste, Italy

E. Tosatti

International Center for Theoretical Physics (ICTP),

Strada Costiera 11, 34104 Trieste, Italy

M. Urbakh $(\bowtie)$

School of Chemistry, Tel Aviv University,

69978 Tel Aviv, Israel

e-mail: urbakh@post.tau.ac.il realized in the laboratories and become predictable by adequate modeling. A discussion of these problems took center stage at the conference Trends in Nanotribology. This conference demonstrated that a substantial progress in understanding the leading factors that determine the frictional response at the nanoscale has been achieved during last decade and indicated the most challenging directions of future research that include: bridging the gap between the nano-, micro- and macroscales in friction, new approaches to control and modify frictional properties and nanomanipulations at interfaces.

This special issue focuses on discussion of microscopic mechanisms of friction and adhesion at the nanoscale and the problem on how to translate the atomistic approaches developed at the nanoscale in the macroscopic world. The contributions cover many important aspects of frictional behavior, including the origin of the stick-slip motion, dependence of measured forces on the material properties, nanomanipulation, effects of thermal fluctuations, surface roughness on the static and kinetic friction and adhesion. The issue aims to provide an overview of current experimental and theoretical works on nanotribology and possible applications. In selecting the papers we have tried to maintain a balance between new results and review-like aspects, so that the present issue is self-contained and, we hope, readily accessible to non-specialists in the field.

Profound thanks go to all our colleagues and friends who have contributed to this special issue. Each has made an effort not only to present recent results in a clear and lucid way, but also to provide an introductory review that helps the reader to understand the different topics. 